\title{
YGdCaVInIG 系的核磁共振研究
}

\author{
乔荣文郭全中李国栋 \\ (中国科学院物理研究所, 北京)
}

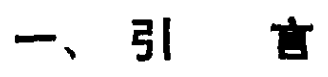

YGdCaVInIG 是一类可得到低饱和磁化强度和低铁磁共振线宽以及低温度系数的新的微 波铁氧体. 最近, 我们实验室已经研究过这一铁氧体系统的磁性、穆斯堡尔谱和铁磁共振. 现 在我们在脉冲高功率 SXP-100 型核磁共振 (NMR) 谱仪上, 用傅里叶变换 (FT) 方法以及 自旋回波 (SE) 法, 分别观测和研究了钎铁石榴石型铁氧体 $\left(\mathrm{Y}_{3} \mathrm{Fe}_{5} \mathrm{O}_{12}\right.$, 简称 $\mathrm{YIG}$ ) 和 YGdCaVInIG 系 $\left(\mathrm{Y}_{1.2} \mathrm{Gd}_{1.2} \mathrm{Ca}_{0.6} \mathrm{~V}_{0.3} \mathrm{In}_{x} \mathrm{Fe}_{4.7-x} \mathrm{O}_{12}, x=0,0.2,0.4\right)$ 多晶材料的不同磁亚点阵中 ${ }^{57} \mathrm{Fe}$ 的 NMR.

测得的 YIG 中 ${ }^{57} \mathrm{Fe}$ 分别在八面体位置和四面体位置处的超精细磁场及其随温度的变 化，与文献上报道的结果 ${ }^{[1]}$ 一致，也与我们实验室用穆斯堡尔效应测得的结果 ${ }^{[2]}$ 相符. 测出的 YGdCaVInIG 系中 ${ }^{57} \mathrm{Fe}$ 的超精细磁场的分布在 380-500 KOe 的范围内, 其中不含 In 的样品 $(x-0)$ 室温下的超精细磁场与穆斯堡尔测量的结果一致。此外, 还测量和讨论了这一样品 的超精细磁场随温度的变化, 以及室温下随 In 含量 $(x)$ 的变化.

\section{二、工作原理及实验方法}

NMR 是以核磁矩作为微探针来研究物质的微观结构的一种磁共振手段. 它的简单原理 是: 当对具有磁性核的物质施加一恒定外磁场 $H_{0}$ 和与 $H_{0}$ 垂直的 射 频场 $h(\omega)$ ，并满足 $H_{0}+H_{10 c}-r \omega$ 条件时 ( $H_{10 c}$ 是核处的局部场, 0 是射频场的角频率), 即产生核磁共振吸 收现象. 这一共振吸收的频率及其线宽等参量, 反映了核周围的微观状态(称为化学环境).强 磁材料中,由于在核处存在一个很强的内禀的超精细磁场 $\left(\text { 约 } 10^{2}-10^{3} \mathrm{KOe}\right)^{[3]}$, 因此不加外恒 磁场只加射频场也能产生 NMR，若已知磁性核的旋磁比 $r$ ，由此即可测量物质内部核处的 超精细磁场。

通常测量 NMR 的方法有连续波法和脉冲法两种,脉冲法又有自由感应衰减 (FID) 法和 自旋回波（SE）法. 由连续波法测得的频(率)域上的吸收谱线与由脉冲法测得的时(间)域上 的 FID 函数互成一对傅里叶变换（FT）关系 ${ }^{[4]}$. 脉冲法比连续波法测量速度快,灵敏度显著 提高. 本文测量采用了脉冲法,实验方框图如图 1 所示.

由于调制脉冲的宽度是有限的 $(\sim 0.5 \mu \mathrm{s})$, 当样品的共振线宽 $\geqslant 1 \mathrm{MHz}$ 时（强磁材料中 共振线宽一般都大于 $10 \mathrm{MHz}$ )，不能采用灵敏度较低的连续波法，也不能用一般的 FID 方法， 而需采用自旋回波（SE）法，这一方法的要点是 ${ }^{[J]}$ : 发射机发射两个间隔为 $\tau$ 的射频脉冲,第 


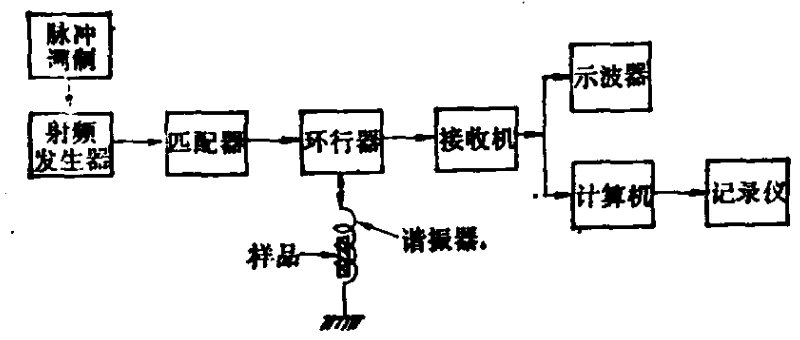

图 1 脉冲法测量 NMR 的实验方框图

一个脉冲使核磁矩旋转 $90^{\circ}$, 称为 $90^{\circ}$ 脉冲,第二个脉冲使核磁矩旋转 $180^{\circ}$, 称为 $180^{\circ}$ 脉冲, 即脉冲序列为: $90^{\circ}-\tau-180^{\circ}$. 在 $180^{\circ}$ 脉冲之后 $\tau$ 时刻处, 出现一个来自样品的回波信号 (自 琁回波). 测量出不同频率下的回波幅度，即可得到自旋回波的频率曲线，由此可测定 NMR 频率。

\section{三、实验结果及讨论}

1. 对 YIG 多晶样品进行的 NMR 测量表明, 由于 YIG 的谱线很窄 (约 $0.2 \mathrm{MHz}$ ), 得到 自旋回波信号后，可直接进行傅里叶变换而得到其共振吸收谱线. YIG 的共振频率和超精细 磁场随温度的变化关系如图 2 所示. 由于 YIG 中 ${ }^{5 /} \mathrm{Fe}$ 核有两种位置,八面体晶位 ( $a$ 晶位)和四 面体昆位 ( $d$ 晶位)，因此存在两种不同的超精细磁场, 从而对应两个共振频率. 曲线( $(a)$ 和 (d) 分别是八面体晶位和四面体晶位的共振频率和超精细磁场随温度 $T$ 的变化关系. 图中还列出

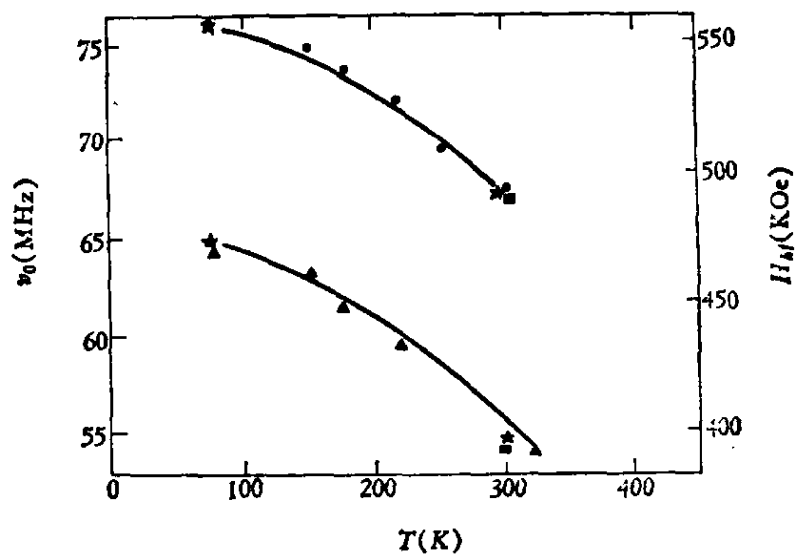

图 2 YIG 中"Fe 核的共振频率 $\nu_{0}$ 和超精细

磁场 $H_{h f}$ 与温度 $T$ 的关系

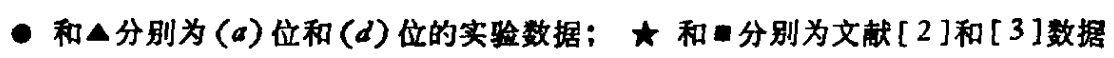

了文献报道由 NMR ( $\star$ ） 和穆斯堡尔效应测量 $(\boldsymbol{a})$ 的数值 ${ }^{[1,2]}$.

由于超精细磁场 $H_{h t}$ 正比于饱和磁化强度 $M_{s}{ }^{[4]}$, 因此 $H_{h}-T$ 的关系就反映了 $M_{s}-T$ 的关系. 由 NMR 可以分别求出两个磁亚点阵上 $M s$ 与 $\mathrm{T}$ 的关系,这是一般磁测量技术所不 能做到的.

2. 对 $\mathrm{Y}_{1.2} \mathrm{Gd}_{1.2} \mathrm{Ca}_{2_{0.6}} \mathrm{~V}_{0.3} \mathrm{In}_{x} \mathrm{Fe}_{4.7-x} \mathrm{O}_{12}$ 系的不同 In 含量 $(x=0,0.2,0.4)$ 样品进行了 NMR 的测量. 由于它们的超精细磁场分布较宽 $(>100 \mathrm{KOe})$, 因此需要采用自旋回波法测量. 测 得的不同样品的自旋回波频谱分布如图 3 所示. 可以看出,与 YIG 相比, 它们含有较多的非 磁性离子, 出现磁性离子的无规分布，因而使它们的超精细磁场分布展宽. 同 YIG 相似， 


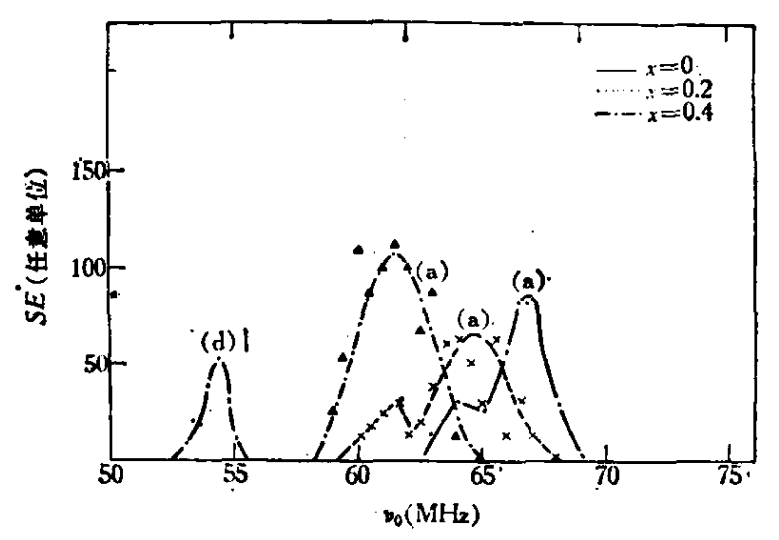

图 $3 \mathrm{Y}_{1,2} \mathrm{Gd}_{1,2} \mathrm{Ca}_{0,6} \mathrm{~V}_{0.3} \mathrm{In}_{\mathbf{x}} \mathrm{Fe}_{4,7-\mathrm{x}} \mathrm{O}_{12}$ 系室温下的自旋回波频谱 $-x=0 ; \cdots \cdots x=0.2 ; \quad \cdot \cdot-x=0.4$

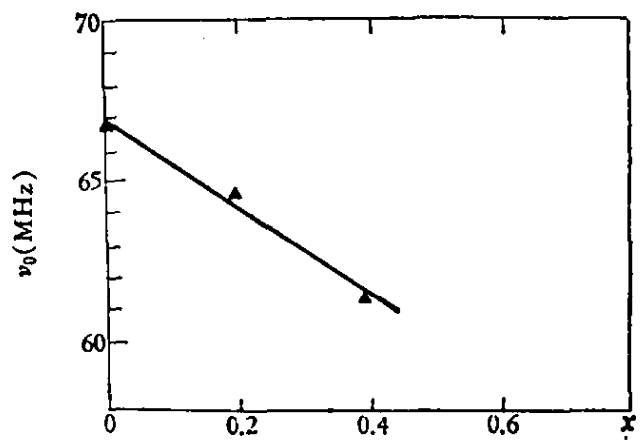

图 4 室温下 $\mathrm{Y}_{1.2} \mathrm{Gd}_{1.2} \mathrm{Ca}_{0.6} \mathrm{~V}_{0.3} \mathrm{In}_{\mathrm{x}} \mathrm{Fe}_{4,7-x} \mathrm{O}_{12}$ 的八面体 (a) 中 " $\mathrm{Fe}$ 的 NMR 频率 $\nu_{0}$ 与 In 含 $x$ 的关系

GdCaVInIG 中 ${ }^{57} \mathrm{Fe}$ 核也有八面体和四面体两种晶位, 因此有两个超精细磁场的分布曲线. $x=0$ 时对应四面体的谱线 $(53 \sim 55 \mathrm{MHz})$ 和对应八面体的谱线 (63-69MHz) 由图 3 可以 看出, $x=0.2$ 和 $x=0.4$ 时对应的四面体晶位的谱线, 由于频率较低需另换测量探头测量. YGdCaVInIG 中,位于十二面体上的 $\mathrm{Gd}^{3+}$ 核也是具有磁性的 $(I-3 / 2)$, 但是由于它的谱线 太弱,需在低温下才能观测到 ${ }^{[6]}$. 测量结果表明,共振频率(谱线的峰值)随 In 含量 $(x)$ 增加 而减少(图 4). $\mathrm{In}^{3+}$ 离子一般占据八面体晶位 $(a)$, 因此 $a$ 位 ${ }^{57} \mathrm{Fe}$ 的最近邻 $(d)$ 不应随 In 含 量变化而变化. 但 $a$ 位 ${ }^{57} \mathrm{Fe}$ 的共振频率随 $x$ 增加而减小的实验事实, 说明在这种铁会体中 $a$ 位 ${ }^{57} \mathrm{Fe}$ 的共振频率不仅与最近邻 ( $d$ 晶位), 而且也与次近邻 ( $a$ 晶位)的磁珄离子浓度有关.

$x=0$ 时,样品的共振频率随温度的变化关系如图 5 所示. 实验发现随着温度降低,频谱 分布曲线变窄变高. 曲线 (a) 和 (d) 分别为 ${ }^{57} \mathrm{Fe}$ 在八面体和四面体晶位的共振频率随温度的 变化关系. 由 NMR 测得的 $x=0$ 时的室温下的超精细磁场为 $484 \mathrm{KOe}$ ( $a$ 晶位) 和 $396 \mathrm{KOe}$ ( $d$ 晶体), 与用穆斯堡尔方法测得的结果相符.

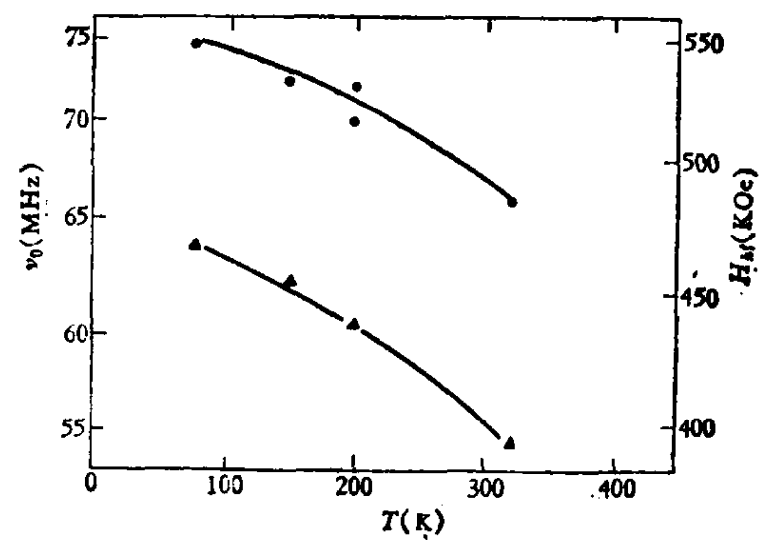

图 $5 \mathrm{Y}_{1,2} \mathrm{Gd}_{1.2} \mathrm{Ca}_{0.6} \mathrm{~V}_{0.3} \mathrm{Fe}_{4.7} \mathrm{O}_{12}$ 中 " $\mathrm{Fe}$ 的核磁共振频率 $\nu_{0}$ 与温度 $T$ 的关系

- 和 $\triangle$ 分别为 $(a)$ 位和 $(d)$ 位的实验数据

YIG 和 $\mathrm{Y}_{1.2} \mathrm{Gd}_{1.2} \mathrm{Ca}_{0.6} \mathrm{~V}_{0.3} \mathrm{Fe}_{4.7} \mathrm{O}_{12}$ 的频率与温度的归一化曲线 $\left(\frac{\nu_{0}(T)}{\nu_{0}(0)}-\frac{T}{\theta_{f}}\right)$ 如图 6 所示. $\nu_{0}(0)$ 为外推 $\mathrm{OK}$ 时的 NMR 频率, $\theta_{f}$ 为居里温度. 由图 6 可以看出, 两种样品的归一化曲 


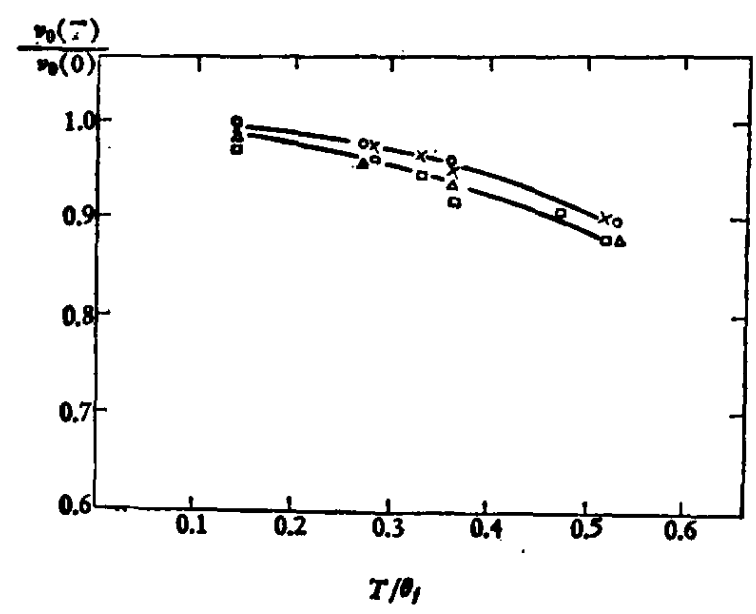

图 6 YIG 和 $\mathrm{Y}_{1,2} \mathrm{Gd}_{1,2} \mathrm{C}_{0.6} \mathrm{~V}_{0.9} \mathrm{Fe}_{4,7} \mathrm{O}_{12}$ 的 NMR 频率与温度的归一化曲线

$X$ 和口为 YIG 的 $a$ 晶位和 $d$ 蜱位的数据； 0 和 $\Delta$ 为 $\mathrm{Y}_{1,2} \mathrm{GN}_{1,2} \mathrm{Ca}_{0,6} \mathrm{~V}_{0,3} \mathrm{Pe}_{4,2} \mathrm{O}_{12}$ 的 $a$ 和 $d$ 晶位的数据

线是重合的,表明了在所测温度区间内稀土离子 $\mathrm{Gd}^{3+}$ 的影响是很小的.

\section{四、结论}

由上述的实验结果和讨论, 可以看出：

1. 当磁性材料的核磁共振 (NMR) 线宽不大时 (如 YIG), 可以由样品的回波信号经傅 里叶变换 (FT) 直接得到 NMR 吸收谱线. 当磁性材料的 NMR 线宽较大时 $(>1 \mathrm{MHz})$, 需 要采用自旋回波法来测定 NMR 频率.

2. 在 YIG 和 $\mathrm{Y}_{1.2} \mathrm{Gd}_{1.2} \mathrm{Ca}_{0.6} \mathrm{~V}_{0.3} \mathrm{Fe}_{4,7} \mathrm{O}_{12}$ 中, $a$ 晶位和 $d$ 晶位的 ${ }^{57} \mathrm{Fe}$ 的 $\mathrm{NMR}$ 频率和超精 细磁场随温度升高而降低. 两种样品的归一化曲线 $\frac{\nu_{0}(T)}{\nu_{0}(0)}-\frac{T}{\theta_{f}}$ 是重合的, 表明在所测量的温 度区间内稀土离子 $\mathrm{Gd}^{3+}$ 的影响是很小的.

3. YGdCaVInIG 中, NMR 频率随 In 含量增加而降低, 表明它与最近邻和次近邻的磁性 离子浓度都有关。

4. 用 NMR 方法测得的 YIG 和 YGdCaVInIG 中 ${ }^{57} \mathrm{Fe}$ 的超精细磁场与由穆斯堡尔方法 测得的结果是相符合的.

致谢: 对张药西、张立和张泮称同志提供实验用样品, 张泮尔和洨启芬同志提供尚未发表的穆斯堡尔数 据及有益的讨论,作者谨表示深切的感谢.

\section{考文 蝴}

[1] Turov, E. A., Petrov, M. P., NMR in Ferro-and Antiferromagnets, 1972.

[2]车国标、徐英庭, 物理学报, 25(1976), 444.

[ 3 ] Watson, R, E., Phys., Rev., 123(1961), 2027.

[4] Müllen, K., Pregoson, P. S., Fourier Transform NMR Techniques, 1976.

[ 5 ] Hahn, E. L., Phys. Rev., 15(1950), 580.

[6] Lo Dand-khoï, Phys. Lett., 28 A(1969), 671. 